\title{
Michel Carmona, Sœur Jeanne des Anges : diabolique ou sainte au temps de Richelieu?
}

André Versaille éditeur, 2011, 334 p.

Daniel Vidal

\section{(2) OpenEdition}

\section{Journals}

Édition électronique

URL : http://journals.openedition.org/assr/23931

DOI : 10.4000/assr.23931

ISSN : $1777-5825$

Éditeur

Éditions de l'EHESS

Édition imprimée

Date de publication : 30 décembre 2012

ISSN : 0335-5985

\section{Référence électronique}

Daniel Vidal, «Michel Carmona, Sœur Jeanne des Anges : diabolique ou sainte au temps de

Richelieu? ", Archives de sciences sociales des religions [En ligne], 160 | octobre-décembre 2012, mis en ligne le 04 septembre 2012, consulté le 21 septembre 2020. URL : http://journals.openedition.org/ assr/23931; DOI : https://doi.org/10.4000/assr.23931

Ce document a été généré automatiquement le 21 septembre 2020

(C) Archives de sciences sociales des religions 


\section{Michel Carmona, Sœur Jeanne des Anges : diabolique ou sainte au temps de Richelieu?}

André Versaille éditeur, 2011, 334 p.

Daniel Vidal

\section{RÉFÉRENCE}

Michel Carmona, Sœur Jeanne des Anges : diabolique ou sainte au temps de Richelieu ?, André Versaille éditeur, 2011, $334 \mathrm{p}$. 

beaucoup écrit, depuis son éclosion au $\mathrm{XVII}^{\mathrm{e}}$ siècle, jusqu'à sa prise en charge aujourd'hui par les historiens des émotions populaires, les exégètes des faits étranges et miraculeux, les chroniqueurs du siècle de Richelieu, les théoriciens de mentalités. Dans cet ensemble foisonnant d'enquêtes et de commentaires, l'ouvrage de Michel de Certeau - La possession de Loudun (Archives/ Julliard, 1970) - a permis une relecture des faits à partir d'une historiographie exigeante, et d'une

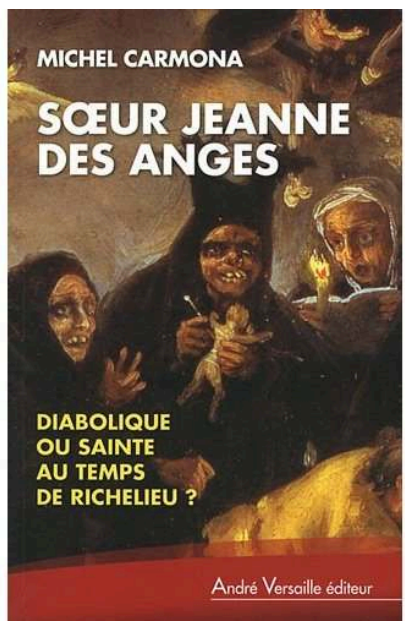
interprétation, désormais irremplaçable, des marges d'une spiritualité mal tempérée. L'analyse du « discours de la possession » (chap. 3), du "théâtre de la possession » (chap. 7), de la "Tératologie de la vérité » (chap.9), par exemple, constituent, et pour longtemps, les références obligées pour toute autre entreprise de déchiffrement. Il fallait en effet en finir avec ce que - dans l'annexe à la Correspondance de Jean-Joseph Surin (DDB, 1966, appendice II, « Jeanne des Anges »), reprise en postface in Sœur Jeanne des Anges, autobiographie d'une hystérique possédée (J.Millon, 1985) - M. de Certeau avait appelé les «reconstructions dramatiques » et les ouvrages « quai de gares », qui ne furent pas l'apanage du seul XIX siècle, mais qui accablèrent également notre $\mathrm{xx}^{\mathrm{e}}$. Heureusement, les œuvres de Robert Mandrou, et notamment Magistrats et sorciers en France au XVII siècle (1968) et l'analyse de psychologie historique qu'il propose ; celle de Robert Muchembled - Sorcières, justice et société au XVI et XVII siècles (1987) et sa comparaison entre culture populaire et culture des élites, ont intégré la possession de Loudun dans une perspective culturelle plus globale, l'événement acquérant un supplément de signification anthropologique. À quoi il convient d'ajouter l'analyse épistémologique et historique de Michel Foucault Histoire de la folie à l'âge classique (1976) - qui inscrit toute «folie » en des âges chaque fois singuliers $\mathrm{du}$ savoir. Cette assignation historique critique qualifiait l'ouvrage précédent de Michel Carmona, Les diables de Loudun. Sorcellerie et politique sous Richelieu, 1988, - tous acteurs rassemblés en une "émotion " collective, son destin tragique inscrit en une séquence historique spécifique.

2 De cette séquence, une figure émerge avec insistance, Jeanne des Anges, supérieure du couvent de Loudun, à qui est consacré le présent ouvrage. À partir des sources archivistiques, des «lettres spirituelles» de diverses mains, des «relations » et "mémoires» contemporains de la possession, de l'Autobiographie de Jeanne, et des "observations» et opuscules publiés au XVII ${ }^{\mathrm{e}}$ siècle,- rien sans doute ne pouvait échapper à l'auteur, des gestes, paroles et faits de Jeanne et de ses consœurs, de la saturation diabolique des corps, de l'intrigue aussitôt qui se noue autour de ces événements d'exception, qui les instrumentalise au plan politique, pour la reconquête des ouailles égarées en Réforme, et pour légitimer, au nom de la lutte contre le Diable, la guerre contre l'hérésie. Jeanne donc, possédée en 1632 au couvent des Ursulines dont elle est la supérieure, et tout ce couvent, ou peu s'en faut, à son tour. Mais qui est Jeanne? Une héritière de ce haut lignage que représente la famille de Belcier, de vocation religieuse aléatoire «partie, écrit-elle, par boutades, partie par persuasion et 
raisons humaines ». Novice à Poitiers, elle est dite dissimulatrice - simulatrice ?" hypocrite ", n'ayant amour que de liberté. À Loudun en 1727, elle en devient très vite la Mère organisatrice et gestionnaire des âmes et des biens. Loudun est foyer de Réforme: "place de sûreté » dans un Poitou bastion du protestantisme. Et creuset d'humanisme: Théophraste Renaudot, Scévole de Sainte-Marthe, y «tiennent salon » où participe parfois un certain Urbain Grandier, curé de st Pierre-du-Marché, de "rhétorique enflammée », rappelait M. de Certeau, faisant référence à l'Oraison funèbre qu'il rédigea à l'occasion de la mort de Sainte-Marthe. Que la possession vienne assiéger les corps des religieuses, et se pose aussitôt la question du coupable. Et que la peste s'étende, voilà qu'à la malédiction des corps faillis s'ajoute, comme un couronnement symbolique, le «mal théologique ».On comprend qu'à l'entremêlement des événements doit se substituer la raison de leur enchainement, de force ou de gré. Les jésuites vont s'y employer, venus à Loudun dès 1607 , et le père Joseph du Tremblay, artisan de l'ascension rapide de Richelieu. Le siège de La Rochelle, puis le rasement du château de Loudun, pour asseoir le pouvoir royal en cette province dissidente, sont, autant que la lutte contre la possession, signes de la puissance conjointe de l'Église et du Roi. Laubardement sera l'acteur éminent jouant au cœur de cette conjonction du religieux et du politique.

3 Jeanne des Anges est assaillie de démons en tous genres, dûment nommés par les exorcistes jusqu'à ce que, longtemps après les premières séances, ils soient expulsés de son corps et de son esprit. Ses démons, ses désirs. M. de Certeau nous met sur la voie, que n'emprunte pas M.Carmona, mais qu'il suggère en accord avec ce que laisse supposer ce qu'en écrit Jeanne. Mais l'aveu d'un désir n'est pas chose facile, sinon par suspicion reportée sur un tiers. Qui désire au fond de moi, qui me désire, de qui suis-je objet de désir? On cerne vite le coupable : Grandier est connu pour son charme et son aisance auprès des femmes. Ce sera donc lui le fauteur de troubles, qui vient posséder les religieuses qu'il visite au creux de la nuit. Il importe peu que cela soit pure invention, que Grandier jusqu'au bout nie toute accusation : deux ans après le début de l'épisode convulsif, il est brûlé vif en place publique. Sans doute ne mettait-il pas trop de zèle à convertir les protestants, et n'obéissait-il pas strictement aux obligations attachées à sa fonction. Cela dut peser lourd dans son inculpation, sous couvert d'un couvent en furie. Et l'on échappe difficilement aux accusations de Jeanne, qui, faisant aveu de son propre désir, condamne par-là même Grandier : "Quand je ne le voyais pas, je brûlais d'amour pour lui, et quand il se présentait à moi, et qu'il voulait me séduire, notre bon Dieu m'en donnait une grande aversion. Ainsi tous mes sentiments changeaient, je le haïssais plus que le Diable, et il m'était si insupportable que je me serais exposée à toutes les furies de l'Enfer plutôt que d'adhérer à la moindre de ses demandes. " Parole de désir, parole funeste qui conduit au bûcher un curé investi de toutes les passions fantasmées d'un cloitre. Mais cette mort n'apaise point Jeanne. Les démons reviennent, les convulsions reprennent, se multiplient, s'organisent en un vaste système de signes qui va très vite se déployer en spectacle où les foules accourent pour faire accueil à cette malédiction qui trouvera en elles son principe de conduction le plus sûr. Ce fut sans doute la raison qui poussèrent politiques et exorcistes à cette mise en spectacle de la possession, assurés qu'ainsi tout un peuple déchu devait être amené à résipiscence. Et Jeanne des Anges, dans l'après-coup du martyre de Grandier, et dans un éclair de lucidité, n'est pas loin de s'estimer victime consentante de ses «endiablements ", à la consternation des exorcistes s'activant autour d'elle. Car ce serait reconnaître la possibilité d'une crise 
venue du plus profond d'une conscience malheureuse. Il faut donc que Jeanne soit mise aux mains d'un confesseur, pour lever toute équivoque.

Jean-Joseph Surin sera cette figure de la " nouvelle spiritualité » jésuite, qui viendra à Loudun en décembre 1734 jusqu'en 1737, pour tenter d'en finir avec cette rage démoniaque qui assiège la supérieure. Il entre ainsi dans la vie de Jeanne comme sa plus forte chance de "guérison " - il en deviendra son tourment le plus radical. Et il faut entendre cela en son sens rigoureux, car Surin, cité ici par Julien Green dans sa préface à l'édition de sa Correspondance par M. de Certeau, est celui qui avait écrit, en juin 1630, «Il est indubitable que qui n'a pas des idées excessives en matière de Dieu, n'en approchera jamais». Et s'approcher de Dieu, vaut ici emprunter des voies insoupçonnées, fussent-elles fréquentées de diables, qui supposent, contre les " énergumènes ", un déploiement d'énergie en excès. Surin voulant "établir en son âme une solide volonté de perfection intérieure ", et s'adressant ainsi au seul registre de la conscience, et du rapatriement en soi de toute culpabilité, Jeanne se dérobe : «Je ne prenais pas plaisir qu'il voulût pénétrer dans mon intérieur ». Mais de son côté, Surin se dispose en telle écoute des spasmes et convulsions de Jeanne, qu'il prend véritablement en charge le "mal», qui sera son propre martyre. J. Green : "Pour vaincre le démon, n'a-t-il pas accepté le risque majeur de pénétrer jusque chez lui, c'est-à-dire dans l'âme de la possédée quitte à partager le sort de celle-ci ? Jeanne des Anges est guérie, mais l'exorciste demeure la proie d'une illusion sinistre: il est damné ». M. de Certeau analysera plus en profondeur cette dérive qui conduit Surin à l'hospice des fous, où il sera violenté, humilié, « avec toutes [ses] passions en émeute ». Ce furent en ces années de lutte contre les "démons "; que Surin écrivit les premières réflexions qui aboutiront ensuite aux deux ouvrages majeurs: Le triomphe de l'amour divin sur les puissances de l'Enfer et Science expérimentale des choses de l'autre vie. Longtemps après la possession de Loudun, qui ne s'achèvera pour l'essentiel qu'au mitan du siècle, Surin, de 1658 à sa mort en 1665, trois mois après la mort de Jeanne, entretiendra une correspondance avec la supérieure de Loudun, où il ne cesse d'inviter Jeanne à une profonde conversion intérieure, à l'oraison attentive, convaincu, note M. Carmona, que «le démon est un ennemi, mais aussi une créature de Dieu». Et que Jeanne, par ces mêmes attaques démoniaques, "expérimente ce qu'est la souffrance", cette voie qui conduit à son Dieu. Et Surin, témoin de ce souffrir, en est le réceptacle et l'aveu: halluciné, pris au piège de sa protégée, au moment où $\mathrm{M}$. Carmona perçoit «la «lente remontée de Jeanne vers la grâce ». Échange funeste ? Transfert et contre-transfert ? En ses lettres, une fois rendu, tel « un mort sortant du sépulcre », à une vie de pleine oblation à Dieu, Surin persiste à inviter Jeanne à prier, se mortifier, afin de trouver en soi le salut, et s'étudier à la pratique "des vraies vertus». Il est vrai que Jeanne, sachant Surin en quelque enfer de l'âme, avait alors souhaité « qu'il plût à Dieu de le mettre dans le vide de la foi, et le purifier de tout le sensible », reversant au compte de l'exorciste la « méthode » qu'il lui avait enseignée.

5 Les chemins, cependant, divergent. Jeanne des Anges «triomphe », écrit M. Carmona. De miracles en miracles, elle fait carrière de sainte et de femme proche du Pouvoir, reçue par Anne d'Autriche, faisant avec Surin pèlerinage à Genève sur le tombeau de François de Sales, et, de retour à Loudun, conversant avec son "saint ange ", lui présentant "les besoins des personnes qui se sont adressées" à elle. "Bureau d'oracles ", précise M. Carmona - qui atteste à la fois de l'apaisement de l'emprise diabolique, et de sa transmutation en "grâces extraordinaires ». Sa mort ne pouvait être qu'édifiante, une fois traversés les temps de possession de l'âme et d'« hystérie » 
du corps, les uns aux autres répondant. Sainte, pourra-t-on dire, parce qu'elle a connu le comble du souffrir et, par l'exhortation de Surin à n'avoir inquiétude que de son "intérieur", elle en a, le plus souvent à contrecoeur, accepté le défi. Sainte, en définitive, parce que possédée.

6 À l'inverse, Surin fut possédé parce que «saint ». Capable d'habiter ces zones d'ombre, parce que tout entier en offrande à son dieu. « Élu-damné », écrivait Julien Green, avant que M. de Certeau n'en donne la raison mystique. Et cette figure crucifiée : «S'il est damné, il ne peut vouloir le bien. Pour obéir à Dieu, il ne peut vouloir que le mal ». La possession de Loudun, où mal et bien se conjuguent en un seul acte subversif, où le diabolique de Jeanne des Anges œuvre à la sainteté de Surin, et celle-ci à la puissance du mal comme volonté de Dieu, constitue, dans l'histoire culturelle du XvII ${ }^{\mathrm{e}}$ siècle, et dans les mêmes décennies où les couvents s'enflamment, à Louviers et Rouen, un passage obligé vers la science de dieu et le savoir de soi, qui l'autorise. 